RAE-IC, Revista de la Asociación Española de Investigación de la Comunicación vol. 8, núm. 15 (2021), 220-241 ISSN 2341-2690

Recibido el 1 de noviembre de 2020 DOI: https://doi.org/10.24137/raeic.8.15.10 Aceptado el 22 de diciembre de 2020

\title{
La mirada de las protagonistas en la investigación de género en comunicación y sus perfiles
}

The gaze of the protagonist in gender research in communication and their profiles

\author{
Izquierdo-Iranzo, Patricia \\ Universidad Rey Juan Carlos (URJC) \\ patricia.iranzo@urjc.es \\ Gómez-Escalonilla, Gloria \\ Universidad Rey Juan Carlos (URJC) \\ gloria.gomezescalonilla@urjc.es \\ Núñez Puente, Sonia \\ Universidad Rey Juan Carlos (URJC) \\ sonia.puente@urjc.es
}

Forma de citar este artículo:

Izquierdo-Iranzo, P., Gómez-Escalonilla, G. y Núñez Puente, S. (2021). La mirada de las protagonistas en la investigación de género en comunicación y sus perfiles. RAE-IC, Revista de la Asociación Española de Investigación de la Comunicación, 8(15), 220-241. https://doi.org/10.24137/raeic.8.15.10 


\section{Resumen:}

Se presenta un trabajo de metainvestigación en Comunicación desde la perspectiva de género fruto de los I+D MapCom1 y MapCom2. En fases anteriores se realizó un retrato (usando análisis de contenido) sobre la investigación en comunicación con la mujer como objeto y/o sujeto, el trabajo presente se realiza desde la perspectiva cualitativa dando voz a sus protagonistas, para ello aplicamos entrevistas en profundidad a las IP de los proyectos I+D en Comunicación sobre género. Como resultados destacan: validación del diagnóstico de desigualdad de género en la investigación en Comunicación; compromiso y sentido comunitario del acto de investigar en género; la dedicación al género presenta intensidades diferentes y un denominador común en la motivación: la identificación personal con la situación de desigualdad de la mujer; las cuestiones de género no siempre se abordan desde la Teoría Feminista; al igual que en la investigación en comunicación genérica, falta mayor transferencia, también se reproducen las técnicas y metodologías aunque varía la predisposición de la mirada hacia el objeto de estudio; no denuncian discriminación explícita en primera persona, pero sí se detecta que abordar el género tiene un coste, se reconoce el avance institucional de las universidades en busca de la igualdad de género.

Palabras clave: estudios de género, brecha de género, metainvestigación, investigación en comunicación, proyectos de investigación I+D, entrevista en profundidad.

\section{Abstract:}

A Communication studies metaresearch work is presented which has been carried out from a gender perspective within the frame of the R \& D Projects MapCom1 and MapCom2. The picture of women as object and subject in media \& communication research previously made using analysis content, it is now reviewed from a qualitative approach by giving the floor to their main figures, for that purpose we use in-depth interviews with the female researchers of R\&D projects about gender and communication. Main results are: they validate the previous picture about gender 
divide in communication research; there is a sense of sisterhood in gender research; they show different levels of dedication to gender research but they all show a common motivation: personal identification with the unequal situation of women; gender issues in communication research are not always approach from the feminist theory anymore; a lack of transfer is detected as it is the case in the no-genderperspective communication research; they use the same researching methods and techniques as in the no-gender-perspective communication research but with a specific approach; personal discrimination has not been reported; it is observed that been dedicated to gender research means an extra effort in terms of more need of explanation in search for comprehension; they report a positive progress in universities in fighting the gender divide.

Keywords: gender studies, gender divide, meta-research, communication studies research, R\&D Projects, in-depth interview.

\section{INTRODUCCIÓN Y ESTADO DE LA CUESTIÓN}

Este trabajo proviene del proyecto: El sistema de investigación en España sobre prácticas sociales de Comunicación. Mapa de Proyectos, Grupos, Líneas, Objetos de estudio y Métodos, (MapCom1), MINECO CSO2013-47933-C4-1P, www.mapcom.es, que elaboró censos de la producción científica en el área de Comunicación; sobre esos censos se realizó una explotación desde la perspectiva de género, y para ello a las variables: tipo de investigación, localización, fecha, contenido y metodología del estudio, se añadieron las variables independientes: Autoría femenina y temática de género y/o mujer. Este cruce permitió dibujar el retrato de la investigación en comunicación realizada por mujeres y/o la investigación en comunicación sobre temática de género, resultados que se describen brevemente en el siguiente párrafo. Este manuscrito presenta como novedad un abordaje cualitativo, para ello se localiza a las IP de los censos, se las entrevista y se analizan sus discursos. 
Principales resultados fase 1: La temática de género $y / 0$ mujer, manifestó comportamientos significativos en relación con las variables cantidad, género de la autoría y carácter del centro, es decir que la investigación presenta rasgos diferenciados cuando toma a la mujer como objeto respecto a esas variables, lo que permite caracterizar la investigación en Comunicación en España sobre temática de género como: muy escasa, únicamente el 4,16\% del total de la investigación en comunicación tiene objetos de estudio de género; de abrumadora autoría femenina, el 97,5\% de esas investigaciones son realizadas por investigadoras, concretamente en las Tesis doctorales sobre cuestiones de género en el 95,2\% de las ocasiones las realizan autoras y en el caso de los proyectos I+D de temas de género el 100\% de las IP son mujeres (Caffarel-Serra, Ortega-Mohedano y Gaitán, 2018, p. 66); también la caracteriza que está fundamentalmente realizada en universidades públicas, ya que esa es la naturaleza de la institución en el 83,33\% de los casos (Martín Algarra, Serrano-Puche y Rebolledo, 2018; Caffarel, Izquierdo-Iranzo y Núñez Puente, 2018). Por otro lado, tenemos que la variable temática de género y/o mujer no ve afectado su comportamiento cuando se cruza con las variables de carácter metodológico: objeto de estudio empleado; técnicas aplicadas; objetivos planteados. La investigación en Comunicación de España tiene mayoritariamente por objeto la comunicación de masas; usa el análisis de contenido y del discurso, y busca la descripción y explicación (Caffarel-Serra, Ortega-Mohedano y Gaitán, 2018). Que la variable temática de género y/o mujer no vea afectado su comportamiento en este cruce significa que cuando se investigan cuestiones de género en el área de Comunicación, el diseño y resto de decisiones metodológicas son parecidas a las tomadas cuando se abordan otros objetos de estudio y, puesto que prácticamente toda la investigación hecha sobre la mujer en comunicación está realizada por autoras, se pudo concluir que, metodológicamente hablando, ellas investigan igual que ellos, incluso cuando lo hacen sobre un tema de/con género (Caffarel, Izquierdo-Iranzo y Núñez Puente, 2018).

En relación con la variable autoría femenina, se obtuvo que la investigación en comunicación en España hecha por mujeres está confinada a las etapas iniciales: se logra paridad en las Tesis Doctorales (mujeres $50,4 \%$ y hombres $49,6 \%$ ), en explotaciones 
posteriores se pudo confirmar que la brecha de género en la presentación de comunicaciones a congresos está superada, pues son más las investigadoras que presentan comunicaciones que los investigadores (Gómez-Escalonilla e Izquierdo Iranzo, 2020), sin embargo, la presencia de la mujer es limitada en la investigación de nivel superior puesto que en proyectos I+D ni un tercio de las IP son mujeres $(29,10 \%)$ (Caffarel, Izquierdo-Iranzo y Núñez Puente, 2018), datos en consonancia con la situación desigual en otras áreas (Lozano e Iglesias, 2014; De Filippo, Sanz y Gómez, 2009; AlcaláCortijo, 2006). La brecha de género en la autoría se agudiza notablemente (7 puntos) si se trata de investigación crítica, donde solo el $22 \%$ de los IP son mujeres frente a un $78 \%$ de varones, incluso en el ámbito de las tesis doctorales se rompe la paridad, donde las autoras constituyen el 38\% mientras que los autores el 62\% (AUTOR, 2018). Este desequilibrio en el liderazgo de la mujer en la investigación en comunicación es un caso más de la dificultad global e histórica que la mujer sufre en el desarrollo profesional, son varios los trabajos que describen esta realidad en el contexto universitario (Martín Algarra, Serrano-Puche y Rebolledo, 2018; De Donoso; Figuera, y Rodríguez-Moreno, 2011, Guil, 2007; Escolano, 2006).

Es a partir de este punto donde comienza la fase de investigación que estamos presentando en este texto y que se enmarca en el MapCom2: Mapas de la investigación en Comunicación en las Universidades españolas de 2007 a 2018 (PGC2018-093358-B100). Es razonable pensar que una mujer que realiza su labor de investigación desde la perspectiva de género sí tiene una conciencia al respecto y desea el cambio social en pro de la igualdad de género, sin embargo, los resultados expuestos hasta ahora no apuntan una dirección clara en este sentido puesto que no se han identificado metodologías propias en la investigación desde la perspectiva de género. Por otro lado, tenemos que hay pocas IP, concretamente 43 , lo que significa que menos de un tercio de la investigación del máximo nivel científico en Comunicación en España tiene una mujer al frente (Caffarel, Izquierdo-Iranzo y Núñez Puente, 2018), y de ellas, muy pocas investigan sobre la mujer, sólo 6 de 43 incluyen el género (como variable principal o transversal), es decir, que solo un $14 \%$ de las IP investigan género. Además, utilizan abordajes semejantes a los empleados cuando investigan otros objetos y metodologías 
similares a las que usan sus compañeros. Esto apunta al arraigo de las tradiciones teóricas, a la reproducción de fórmulas metodológicas en un campo de conocimiento relativamente joven y a la influencia de la comunidad científica. La falta de equilibrio de género en el liderazgo de la investigación, en definitiva, suma otro agujero negro (Giménez-Toledo y Jiménez-Contreras, 2013) al conjunto de debilidades existentes en nuestro campo.

Dado este escenario realizamos un abordaje netamente cualitativo, aplicando técnicas conversacionales a las IP de los proyectos I+D sobre temática femenina y así profundizar en los objetivos que persigue la mujer que investiga sobre la mujer en el área de la Comunicación y en qué circunstancias lo hacen. Esta segunda fase ya presentó sus principales resultados en el V Congreso Nacional TMIC-AEIC. Investigar la comunicación en y desde la periferia, que tuvo lugar en la Facultad de Comunicación y Documentación de la Universidad de Murcia entre el 21 y el 22 de noviembre de 2019 con una comunicación titulada: La investigación de género en comunicación. De los números a las palabras. Esos mismos resultados se exponen ahora de manera más extensa y pausada, aprovechando las ventajas que el artículo como vehículo de difusión permite frente a las limitaciones de las reuniones científicas en ese sentido.

\section{METODOLOGÍA}

El abordaje cuantitativo previo - análisis de contenido- (Izquierdo-Iranzo, GaitánMoya y Caffarel-Serra, 2020), nos permitió describir a la mujer como sujeto y como objeto en la investigación en Comunicación, para esta nueva etapa planteamos una metodología de corte cualitativo porque buscamos ahondar en la comprensión del fenómeno a partir de la voz de sus protagonistas, es decir, añadimos el punto de vista de las propias investigadoras a la pregunta de: ¿Cómo investiga la mujer cuando investiga sobre la mujer en Comunicación?

La construcción de la muestra parte del siguiente universo: el total de proyectos I+D realizados en España sobre prácticas sociales de Comunicación desde 2007, cuyo censo 
se elaboró en MapCom1(CSO2013-47933-C4-1P), esto es: $144^{1}$ proyectos, 43 dirigidos por mujeres (30\%), y de estos solo 6 tienen objetos de estudio con perspectiva de género; 6 proyectos del total de 144 significa que solo un 4,16\% de los I+D en Comunicación se preocupan por el género de manera protagónica. Por tanto, nuestro universo lo componen esas 6 investigadoras cuyos trabajos versan sobre: las relaciones de género en diversas superficies de comunicación; la representación de la mujer en algún producto mediático o los usos y prácticas comunicativas de grupos femeninos concretos. La ventaja de abordar una población tan específica y restringida es su accesibilidad, de hecho, el $100 \%$ del universo que representa el fenómeno abordado participó en la investigación.

Respecto al tiempo que cubre esta investigación es necesaria la siguiente aclaración: el I+D MapCom1 tiene lugar entre 2014-2017 y estudia cómo se realiza la investigación en comunicación entre 2007 y 2013. Los sujetos investigados forman parte de esta muestra por haber sido las responsables de I+D censados entre 2007 y 2013, esto es, proyectos concedidos en esa ventana temporal pero su ejecución ha podido extenderse durante los años siguientes, y su opinión se recoge en 2019, ya dentro del I+D de continuación MapCom2: Mapas de la investigación en Comunicación en las Universidades españolas de 2007 a 2018 (PGC2018-093358-B-I00). Estos saltos en el tiempo pueden generar una sensación de discontinuidad o incoherencia, pero son inevitables en los estudios longitudinales que además trocean sus acotaciones temporales para adecuarse a las convocatorias de financiación. No obstante, todas las entrevistadas han seguido en activo, por lo que sus discursos de hoy recogen todo el marco temporal retrospectivo.

Los datos fueron recogidos mediante entrevistas en profundidad, una técnica cada vez más en desuso debido a las actuales lógicas productivas de la ciencia, pero singularmente adecuada dados los objetivos de esta investigación, ya que se trata de una entrevista no directiva y centrada, es decir, monográfica a propósito del propio entrevistado (Gaitán y Piñuel, 1998, p. 94). Al igual que sucede en otras técnicas de

\footnotetext{
${ }^{1}$ MapCom mantiene sus censos abiertos porque si bien el trabajo está concluido, puntualmente se puede seguir identificando algún nuevo I+D, por ello, esta cifra puede ir sufriendo pequeñas variaciones en los sucesivos trabajos que se van realizando y que cada vez toman el dato actualizado.
} 
marcado carácter personal e individual, como los métodos autobiográficos o las historias de vida, en las entrevistas en profundidad la relación del investigador con el sujeto es diferente, ya que la conversación pausada permite una mayor confianza y previsiblemente una mejor explicación-comprensión del fenómeno en estudio. Al caracterizarse también por estar escasamente precodificada no se desarrolló un guion exhaustivo, pero sí se perfilaron puntos que debían tratarse, tales como, la opinión sobre el diagnóstico que arrojó el MapCom1, el motivo de abordar estudios de género, la opinión sobre si los métodos en la investigación de género deben ser diferentes al resto de áreas dentro de las Ciencias Sociales o si encuentran resistencias internas y/o externas. Los encuentros se realizaron tanto presencialmente como en remoto y aunque tuvieron una duración variable, todos se enmarcaron entre los 45-60 minutos.

\section{ANÁLISIS Y RESULTADOS}

En este apartado analizamos los discursos de las 6 IP del censo de MapCom que han realizado proyectos de género, aportan su opinión acerca de la situación de las investigadoras en comunicación y de la investigación en comunicación que aborda objetos de género partiendo de su propia experiencia. Comenzamos indicando que los 6 testimonios fueron 6 discursos marcadamente unipersonales, lo que significa que además de la investigadora pudimos llegar a la persona, metodológicamente hablando esta "riqueza de datos" nos ha permitido responder las preguntas de investigación planteadas.

\subsection{VALORACIÓN GLOBAL DE LA SITUACIÓN Y VINCULACIÓN CON EL OBJETO}

Pedida su valoración sobre el diagnóstico que arroja el MapCom1 (investigación de alto nivel en Comunicación dominada por hombres, por tanto, se estudia poco sobre género) encontramos unanimidad, siendo "mucho" y "plenamente" las valoraciones expresadas más veces. El testimonio "Existe segregación vertical y horizontal en la docencia, en la gestión y también en la investigación, y eso hace que quienes lideran la investigación sean mayoritariamente hombres" (TG) apunta causas orgánicas, la misma voz añade motivos de otra naturaleza que apuntan a un sentido de filiación endogrupal: "Es lógico que la investigación de lo femenino esté liderada por mujeres, pero también se debería 
abrir a hombres, igual que espacios que son solo de hombres también se debería abrir a mujeres", en este mismo sentido también obtuvimos: "quizá para ciertos temas nos gusta trabajar más con mujeres" (CL) y "el hecho de ser mujeres hace que haya una mayor empatía y un mayor compromiso" (AHR). El testimonio de que el diagnóstico alcanzado en el MapCom 1 es "preocupante pero cierto" (CE), sitúa la opinión general respecto a este primer punto de manera clara y concisa.

Respecto a la vinculación con el tema, encontramos las siguientes respuestas: una persona manifestó que el I+D sobre género fue algo puntual, para el resto de sujetos sí es una constante en su trabajo con mayor o menor intensidad, 2 de las investigadoras usan el género como línea transversal a estudios sobre narrativas formando parte del estudio de la alteridad, y para 3 de ellas esta es su única línea de investigación, estas 3 autoras manifiestan reconocerse plenamente en la Teoría Feminista, una afirma: "no entiendo que se pueda hacer investigación sin género" (CE).

A esta explicación de la vinculación con el tema, desde la trayectoria curricular, cabe sumar otra de carácter más personal que aparece recurrentemente en diversos puntos de sus discursos y en la que sí encontramos homogeneidad, todas manifiestan un compromiso personal con las cuestiones de género y en algún caso se habla también de compromiso emocional; esta coincidencia en el reconocimiento de un vínculo con el tema no sorprende ya que se señala como característica inherente "creo que es lo que define esta línea" (NP). Al compromiso personal se le da salida vía académica, a su vez reconocen que la aproximación científica constituye un refuerzo de la inquietud personal.

La vinculación con el tema se relaciona con otras dos cuestiones abordadas muy similares entre sí: Motivo por el que eligen temas de género y Manera de definir los temas. Además del compromiso personal señalado se añaden causas propias de la lógica universitaria como el vacío científico existente en España al respecto (indicado por la mitad de ellas), o ser el eje vertebrador para construir equipos que aglutinen perfiles diversos. En dos casos la identificación con el feminismo se sitúa a nivel vital antes de la llegada a la vida universitaria como estudiante, encontrando en la academia un espacio 
donde vehicularlo, un testimonio señala que "hacer la tesis sobre feminismo fue un acto de justicia poética" (CE).

En la manera de definir los objetos de estudio sobre género, solo en un caso decía explícitamente responder a los CFP, en el resto parece ser fruto de la mirada sobre el entorno y sobre una misma: "desde este interés inicial como espectadora me empiezan a surgir preguntas" (MM); "como mujer, claro que los temas de mujer me interesan y afectan especialmente" (AHR); "me alarma que constantemente se estén desacreditando algunas identidades, una buena parte del discurso de odio es contra las mujeres" (CL), "parto de la práctica, cada vez más de la experiencia biográfica” (CE).

Es decir, hoy día todas manifiestan sensibilidad y compromiso personal hacia la situación de desigualdad de la mujer y lo aplican en su labor investigadora, se detecta que quienes tienen esa identificación desde etapas preuniversitarias, su canalización en la academia es más intensa y a su vez influye más en su visión como ciudadana. Sea como sea lo relevante es que: "es consustancial a los estudios de género, siempre vamos a intentar mejorar aquella situación que estamos analizando (...) establecer evidencias (...) eso es contribuir a que se haga realidad" (AHR), y eso sin duda lo logran todas y cada una de ellas desde el momento que consiguen elevar al máximo nivel de la creación científica las cuestiones de género.

La opinión de las entrevistadas respecto al estadio de los estudios de género en nuestro país ya se aludió y contestó parcialmente previamente cuando varias de ellas expresaron que uno de los motivos por el que dedicarse a esta línea es precisamente el vacío existente. Ahora precisan cómo se concreta ese "vacío" y además de manera polifónica porque las respuestas abarcan todo el espectro posible. En un extremo tenemos quien opina que ese estadio es tan deficitario que de hecho no hay un ámbito disciplinar de género, y en el otro quien opina que esta línea sí está asentada en nuestro país; entre un polo y otro, otras opiniones hablan de rápida institucionalización, pero carente de reflexión crítica; de avance, pero a falta de crecimiento y visibilización; o de gran impulso en el último decenio. Atendiendo al discurso conjunto de cada una de ellas se puede observar que sí manifiestan una idea común: "se ha avanzado", a la que se añade el 
matiz "pero falta camino" expresado en variados niveles de optimismo/esperanza. Las posturas más escépticas y críticas se corresponden con los perfiles que desde un principio se han reconocido plenamente en la Teoría feminista, no es ilógico pensar que quien tenga mayor identificación y esté más permeado por unos postulados abrigue mayores expectativas en su desarrollo teórico.

\subsection{OPINIÓN SOBRE LA METODOLOGÍA EN LA INVESTIGACIÓN DE GÉNERO, SU POTENCIAL TRANSFORMADOR Y LA TRANSFERENCIA EFECTIVA}

Cuando conversamos acerca de la metodología en la investigación de género y particularmente en las investigaciones que han realizado ellas, encontramos lo siguiente: en la enumeración de las técnicas empleadas se repiten varias veces las mismas: historias de vida, grupos de discusión, análisis de contenido, encuestas, entrevistas, observación participante, análisis etnográfico... en definitiva se indican las técnicas habituales en las Ciencias Sociales, y es que como afirma una de las IP, "en la metodología en todas las Ciencias Sociales, seguimos siendo del siglo XIX, usamos los mismo" (CE). Los matices se presentan a la hora de dilucidar cómo debiera ser la metodología empleada, podemos agrupar las opiniones al respecto en dos conjuntos: en uno señalan que emplean el mismo abordaje metodológico en toda la investigación que hacen, bien por ser deudoras de una tradición teórico-metodológico determinada, bien porque la replicación de estudios lo demanda. Digamos que responde a una inercia y un modus operandi asentado en su quehacer investigador, lo cual no significa que se haga inconscientemente, al menos en uno de los casos se expresa que es algo consciente y se defiende que el marco metodológico no debe cambiar por que cambie el objeto de estudio.

En el otro grupo sí se apuesta por la diferenciación, los tres testimonios de este grupo inciden mucho en la mirada, como el factor diferencial de lo que debe ser una investigación hecha con perspectiva de género, a lo que también se suma la actitud: "menos investigar sobre y más investigar con, (...) investigar desde la metodología Feminista es ponerme en una disposición particular de diálogo con el otro" (CE), en este mismo sentido encontramos: "se empieza a hacer una reflexión sobre lo importante que 
es no solo el objeto, sino el sujeto de la investigación" (NP) refiriéndose tanto a las personas investigadas como a las que hacen la investigación, a lo que también cabe añadir el contexto como concepto clave de esa caracterización propia de la investigación en género. Además, se opina que de estos aspectos diferenciadores dependerá el alcance transformador de la investigación realizada, aunque otra participante cuestiona si son ellas a quienes corresponde llevar a cabo esos cambios. Respecto a la idea de transformación, también encontramos: “Mayor responsabilidad transformadora por coherencia teórica (TG)", apelando al origen de los estudios de género en comunicación vinculados a los estudios culturales, a la Teoría feminista y la orientación crítica de estas corrientes que buscan no solo comprender una realidad sino también transformarla. Los tres perfiles de este segundo grupo que apelan a una personalidad metodológica propia y distintiva son los tres perfiles que se dedican exclusivamente a los estudios de género.

Analizamos ahora la posibilidad de convertir el potencial transformador que va en el ADN del feminismo, en transferencia en pro de la igualdad de género en la sociedad. Independientemente de cómo se articule su abordaje, todas coinciden en señalar que la naturaleza de lo investigado implica mayor intención de intervención, incluso se afirma: "Ios verdaderos resultados tienen que ser esos, los que tienen que ver con la transferencia" (MM). Uno de los testimonios insiste en que esta dimensión no está reñida con la calidad en producción académica: "intentamos la intervención sin restar validez científica" (NP). Existe una conciencia común como investigadoras en avanzar más allá de la creación de conocimiento, se ponen ejemplos de diferentes esferas donde intervenir: leyes, contenidos mediáticos, actividades escolares etc., y se afirma: "Ahora estamos en el postfeminismo, es decir, que el avance no tiene que ir de la mano de lo político (...) El contenido científico ha llegado a la sociedad y a la profesión" (TG), además todas manifiestan "transferir" el valor de la igualdad de género en las aulas, pero solo una narra experiencias concretas llevadas a cabo para promover la mirada de género en el desempeño profesional de los estudiantes de Comunicación a través de actividades con la población de la comunidad local, justamente se trata de un perfil que no investiga exclusivamente en género, por tanto la transferencia empírica no se relaciona directamente con los perfiles que más se basan en la Teoría Feminista. 
Todo lo analizado nos permite esbozar unos perfiles de las investigadoras en los que se relacionan el tipo de identificación con la temática de género con la extensión de su acción:

Tabla 1. Perfiles de las investigadoras

\begin{tabular}{|l|c|c|c|c|}
\hline & $\begin{array}{c}\text { Realiza } \\
\text { investigación } \\
\text { desde la } \\
\text { perspectiva de } \\
\text { género }\end{array}$ & $\begin{array}{c}\text { Identificación } \\
\text { con cuestiones } \\
\text { de género } \\
\text { desde el plano } \\
\text { vital } \\
\text { (sensibilidad } \\
\text { personal) }\end{array}$ & $\begin{array}{c}\text { La inquietud } \\
\text { por el género } \\
\text { impregna el } \\
\text { abordaje } \\
\text { metodológico } \\
\text { (sensibilidad } \\
\text { científica) }\end{array}$ & $\begin{array}{c}\text { Extiende su } \\
\text { acción } \\
\text { científica a lo } \\
\text { sociopolítico } \\
\text { (sensibilidad } \\
\text { ciudadana) }\end{array}$ \\
\hline $\begin{array}{l}\text { Quien basa en la Tá } \\
\text { feminista su } \\
\text { mirada global }\end{array}$ & Exclusivamente & Sí & Sí & Sí \\
\hline $\begin{array}{l}\text { Quien imbrica } \\
\text { inquietudes } \\
\text { personales y } \\
\text { científicas }\end{array}$ & Regularmente & Sí & Sí, de forma \\
más & A veces \\
\hline $\begin{array}{l}\text { Quien tiene una } \\
\text { relación coyuntural } \\
\text { con la } \\
\text { investigación en } \\
\text { género }\end{array}$ & Puntualmente & Sí & A veces & No \\
\hline
\end{tabular}

Fuente: Elaboración propia

\subsection{VALORACIÓN Y PRESTIGIO DE LAS INVESTIGADORAS DE GÉNERO}

Nuevamente encontramos consenso y disenso parcial en los discursos, en este caso respecto al lugar de las investigadoras en la comunidad científica y, concretamente, de las investigadoras sobre género. El titular del diagnóstico sí es unánime y lo resume la frase: "iEs tan obvio que a medida que avanzamos en nivel formativo y jerarquía, el embudo nos va dejando abajo!" (MM), pero existen matices en la visión de cómo se materializa esa desigualdad. Cuatro explícitamente y una implícitamente, manifiestan no haber padecido discriminación en el sentido de percibir que dedicarse a cuestiones de género, o los trabajos realizados en esta línea, hayan mermado su labor. Cuatro de ellas sí declaran conocer discriminación de manera indirecta al haberla escuchado por boca de otras colegas que relatan resistencias en la aceptación/valoración de su trabajo.

En este punto conviene distinguir entre la valoración como prestigio (reconocimiento dentro y fuera de la academia, status intelectual, peso del área...) y la valoración como 
evaluación que permite o veta el avance en la carrera profesional. Referida a esta última acepción, ninguna expresa haber padecido frenos explícitos, por ejemplo, en la concesión de los I+D. Respecto a las oportunidades para publicar todas relatan estrategias para esquivar posibles dificultades, tales como la selección de las revistas más adecuadas, práctica habitual y lógica en toda la investigación, en más de una ocasión se indica el aumento de journals receptivos al abordaje de y desde el género en los últimos años. Sí hay un testimonio muy clarificador al respecto: "La propia ciencia veta porque no contempla la acción política y transformadora, y si lo hace es con más resistencias que en otras áreas como infancia, educación..." (NP), quien continúa: "Tiene apoyo formal pero no real, en los I+D sí hay expertos, pero no en sexenios, acreditaciones... lo publicado sobre género tiene menos probabilidades porque no hay expertos". Referido a la primera, la acepción de valoración como prestigio, la mitad indica haber percibido lastres que rodean la materia y que van desde considerarse algo menos sesudo, hasta tildarse de parcial y sesgado por parte de otros colegas.

En una valoración de la desigualdad referida al funcionamiento del día a día en la universidad solo una de las entrevistadas manifiesta haber encontrado a título personal resistencias en el entorno laboral inmediato, el resto coincide en señalar que no las ha encontrado, y si así ha sido ha podido neutralizarlas: "En alguna ocasión he tenido que escuchar algún comentario de algún colega con poca gracia, pero te aseguro que los corto radical e inmediatamente" (CL); no han encontrado problemas ni por parte de colegas ni de superiores, tampoco en la estructura oficial o administrativa. Incluso en 4 ocasiones se indica que en el marco de la institución de pertenencia, el tema igualdad de género ha tenido crecimiento por diversas vías: creciente interés como tema de estudio por parte de colegas y creación de equipos, implantación de estudios oficiales, inauguración de unidades específicas, puesta en marcha de planes de igualdad... Junto a estos positivos ejemplos todas reconocen que el ámbito académico es una burbuja privilegiada respecto a otros ámbitos laborales en los que las micro y macro resistencias afloran con mayor asiduidad. También es unánime señalar la positiva acogida del alumnado sobre estos temas de forma creciente y natural. 
Acabamos el epígrafe de análisis con unas últimas reflexiones que nos dejan un variopinto retrato del tema y, que por ser de tinte más personal, en esta ocasión vamos a dejar en el anonimato:

"Mi entorno cercano fuera de la universidad me percibe como monótona con relación a esto"; "Me da miedo que se pueda hacer populismo con estos temas porque están en boga"; "Funciona la Teoría del etiquetaje, si eres la feminista, como que lo que dices vale menos y esas micro resistencias están todos los días"; "La mejor manera de intervenir entonces es desde el prestigio, desde la máxima calidad JCR Q1, nadie lo cuestiona"; "Parece que estás más satisfecho cuando te implicas en estos estudios".

\section{DISCUSIÓN Y CONCLUSIONES}

Toda la muestra está de acuerdo con el diagnóstico alcanzado en la fase previa de la investigación (MapCom1), donde se ve que la investigación de género en comunicación solo le interesa realizarla a la mujer y, debido al techo de cristal existente (Guil, 2007; Escolano 2007; De Donoso, Figuera y Rodríguez-Moreno, 2011) eso hace que este tipo de investigación sea escasa y quede fundamentalmente restringida a los primeros niveles de la carrera académica. La unánime validación de este retrato desde la experiencia de las 6 voces constituye un aporte científico más al respecto. Los discursos de las investigadoras nos permiten explorar otros factores que caracterizan el fenómeno, uno de ellos es el sentido identitario y de comunidad que juega el rol de género, es un nos gusta investigar juntas e investigarnos, nos preguntamos si esto supone una deriva endogámica del sentido de pertenencia grupal o se da para cubrir el vacío y desafección existentes, lo que a su vez nos lleva a cuestionarnos si es positivo o negativo. Para ello, recurrimos a la literatura científica sobre el concepto de sisterhood (=hermandad), clave durante la segunda ola del feminismo al aportar el sentido de unión mediante la solidaridad y los objetivos comunes, y la noción de horizontalidad en las relaciones, idea reiteradamente referida en los discursos de las entrevistadas. El concepto de sisterhood se revisa en la tercera ola, cuando la figura del hombre se empieza a ver como clave para el crecimiento del movimiento y sus objetivos. Sin embargo, para autoras como Astrid Henry (2004), el rechazo a este y otros conceptos 
que distancia a la 2a y $3^{a}$ ola, menoscaba el poder de la acción colectiva. La realidad es que en la actualidad dentro del movimiento feminista la idea de sisterhood sigue en debate entre quienes cuestionan la figura del hombre dentro del movimiento feminista y quienes la consideran necesaria, por eso Evans (2015) se plantea si el concepto de sisterhood es todavía posible o deseable. En los discursos de nuestras investigadoras solo en un caso se entra en este tipo de disquisiciones, cuando invita a abrir la investigación de lo femenino a los colegas masculinos, en el resto de los testimonios simplemente se evidencia la realidad respecto al hecho concreto investigado: quienes investigan sobre mujeres son mujeres, también se expresa en más de un caso la confortabilidad que produce compartir género y trabajo. Por tanto, la pregunta queda sin responder, no es algo ni negativo ni positivo, la abrumadora presencia de investigadoras frente a investigadores en cuestiones de género es simplemente un rasgo más que define la investigación en/sobre género. Sin embargo, la observación del fenómeno permite afirmar que es probable que se esté produciendo una transición y que los colegas masculinos empiezan a participar en esta línea de investigación, quedamos atentas al comportamiento de la variable sexo de investigadores sobre género, dentro de la línea de metainvestigación en Comunicación desde la perspectiva de género.

Para la mayoría de las entrevistadas ( 5 de 6 ) el tema género es una constante en su trabajo, esta elección presenta 3 grados diferentes de vinculación en la trayectoria curricular: puntual, parcial y plena, y en el plano personal todas se sienten concernidas ante la situación desigual de la mujer. Sin embrago, sólo la mitad desempeña su labor científica desde la Teoría Feminista, es decir, desde la perspectiva más crítica. Se observa que cuanto más temprano en edad es el vínculo con las cuestiones de género, más sensorial es la relación que se establece con el tema, se tiene una visión más crítica y una proyección académica y sociopolítica más intensa. También hay otro camino: quienes observan el entorno, se sienten concernidas y le ponen la lupa científica. Dicho coloquialmente es como si a algunas de ellas la inquietud les saliera de dentro a fuera, y a otras la motivación les llegara de fuera hacia dentro, podemos decir que la motivación e inquietud de las investigadoras en comunicación por abordar temas de 
género puede ser centrífuga o centrípeta. No importa si las trayectorias son más pioneras o más circunstanciales, más teóricas o más prácticas, si arrancan desde lo emocional, lo personal o lo pragmático, si hay exclusividad en la dedicación o no, si hay convicción endógena "autodidacta" o influencia del entorno, lo relevante es que todas ellas contribuyen a que el género esté en la agenda de la investigación con el esfuerzo que ello implica (Durán-Bellonch e Ion, 2014) y al hacerlo con su personalidad propia, contribuyen a la naturalización del hecho, lo cual se perfila como un interesante antídoto contra las voces que dentro y fuera de la academia ven como sesgados los estudios de género.

El modelo fuerza centrífuga, donde la vocación temprana (y pasional) se fusiona con la investigadora que ejerce una dedicada carrera al género, y el modelo fuerza centrípeta en el que la investigadora en etapas más maduras imbrica parte de su actividad científica y su mirada personal al género, no son estancos, de hecho, las 6 investigadoras transitan entre ambos desplazándose cada una de ellas a lo largo de diferentes puntos. La fuerza centrípeta y la centrífuga son complementarias y quizá nos habla del comienzo de un periodo de ciencia normal (Kuhn, 1989) respecto a la cuestión que nos ocupa. El género como sujeto y objeto de estudio es ese centro, que tomamos como referencia para describir los movimientos de las investigadoras dentro de su órbita.

El principal resultado con relación al uso (o existencia) de metodologías propias en los estudios de género en comunicación es que no todas las investigadoras aprueban la necesidad de metodologías propias en este campo. Todas manifiestan emplear las técnicas habituales en el conjunto de las ciencias sociales, tanto de corte cuantitativo como cualitativo, hecho que confirma el dato obtenido por MapCom1 de que la variable autoría femenina se comporta igual en su cruce con empleo de técnicas y métodos de investigación. Quienes ponen el énfasis en la diferenciación indicen y coinciden en señalar la mirada y la actitud como los factores diferenciales. En quienes no ven la necesidad (o no aplican) cambios en el registro metodológico observamos a lo largo del discurso que la predisposición sí varía, por lo que concluimos que también están adaptando la forma de investigar. No son las herramientas sino la manera de usarlas. 
Respecto a la metodología conviene aclarar que la reiteradamente expresada identificación personal con el tema, o incluso este ajuste en técnicas y métodos, no compromete la objetividad científica sino todo lo contrario. Estamos en Ciencias Sociales y Humanísticas, no estamos en Ciencias Exactas y Naturales, nuestras respuestas no son únicas ni conocidas de antemano, la validez de nuestras respuestas depende de la capacidad de las investigadoras de permearse con el sujeto y el objeto investigado, nuestra propia condición actante en el contexto social nos convierte en la mejor técnica-instrumento-modo de conocer lo que estudiamos, no nos condiciona, sino que nos habilita.

En el $A D N$ del feminismo y las preocupaciones por la igualdad de género está el anhelo de transformación, pero como siempre que tratamos de medir efectos resulta algo escurridizo, en este caso además porque incluye muchos más aspectos vitales de los que se pueden recoger en una entrevista, -la propia técnica de recogida de datos o su posterior tratamiento ha podido ser insuficiente-, el dato es que solo una de las participantes explicita acciones de transferencia (primer paso para la transformación) con el entorno fuera de la universidad. Esto significa que se debe reforzar esta tarea en nuestras áreas no solo personal sino también institucionalmente, quizá el recién inaugurado sexenio de transferencia constituya un aliciente en este sentido.

El relato concreto del día a día en su profesión y en relación con su dedicación a la investigación con género, parece retratar un escenario más positivo que el diagnóstico sobre el estado de la investigación con género en comunicación del que partimos, la diferencia de años entre lo uno y lo otro (cfrs. El apartado Metodología) nos invita a pensar en una evolución positiva del asunto fruto del paso del tiempo. Ninguna ha visto limitada su carrera por este motivo, y la mayoría relatan una evolución en la igualdad de género en sus instituciones de pertenencia materializado en acciones visibles. Parece que cuanto más concreta y cercana es una esfera (mi propia carrera, mi alumnado, mi departamento...), más se percibe la evolución de la igualdad, no así cuando el tema se aborda en toda su complejidad social "en estos tiempos de polarización (...) se ocupa políticamente el espacio de la víctima" (NP). En definitiva, se reconocen avances a la par 
que se asume la tarea pendiente ante el históricamente residual papel de la mujer en la ciencia.

Concluimos reflexionando sobre la pregunta de investigación original: ¿hasta qué punto buscan las autoras, de manera consciente, que su trabajo científico sobre la mujer incida en la situación de la mujer en la sociedad actual? Sus discursos dejan evidencia de un compromiso notorio y un quehacer real, si la ciencia no es suficiente para solucionar problemas sociales, habrá que buscar más causas, nuevas alianzas y desde luego, seguir trabajando desde la ciencia.

\section{AGRADECIMIENTOS}

Agradecimiento sincero a las 6 investigadoras que generosamente nos dedicaron parte de ese tiempo que en la bolsa de valores de la vida actual tanta cotiza, pero sobre todo reconocimiento a las 6 mujeres que compartieron su sabiduría e inquietudes personales con nosotras. Ojalá hayamos sabido plasmar correctamente el sentido de vuestras palabras.

\section{REFERENCIAS BIBLIOGRÁFICAS}

Alcalá-Cortijo, P. (2006). A ras de suelo. Situación de las mujeres en las instituciones científicas. Ciencia, tecnología y género en Iberoamérica. Monografías 29. Madrid: CSIC.

Banet-Weiser, Sarah (2018). Empowered: popular feminism and popular misogyny. Durham: Duke University Press. Recuperado de http://eprints.Ise.ac.uk/89198/ Cañibano, C., Fox, M. F. y Otamendi, F. J. (2016). Gender and patterns of temporary mobility among researchers. Science and Public Policy, 43(3), 320-331.

Caffarel Serra, C., Izquierdo Iranzo, P. y Núñez Puente, S. (2018). ¿Cómo investiga la mujer cuando investiga sobre la mujer en comunicación?. En E. Martínez Rodrigo (Coord.), Propuestas de investigación en áreas de vanguardia (pp. 73-86). Madrid: Tecnos. 
Caffarel-Serra, C., Ortega-Mohedano, F. y Gaitán, J. (2018). Communication research in Spain: Weaknesses, threats, strengths and opportunities. [La investigación en comunicación en España: Debilidades, amenazas, fortalezas y oportunidades]. Comunicar, 56, 61-70. https://doi.org/10.3916/C56-2018-06

Colás-Bravo, Pilar (2008). Género y Ciencia. Líneas y metodologías de investigación. En C. Jiménez Fernández y M. G. Pérez Serrano (Coords.). Educación y género. El conocimiento invisible (pp. 191-216). Valencia: Tirant lo Blanch.

de Donoso, T., Figuera, P. y Rodríguez-Moreno, M. L. (2011). Barreras de género en el desarrollo profesional de la mujer universitaria. Revista de Educación, 355, 187-212. Recuperado de http://www.educacionyfp.gob.es/revista-de-educacion/numerosrevista-educacion/numeros-anteriores/2011/re355/re355-08.html

de Filippo, D., Sanz Casado, E. y Gómez, I. (2009). Movilidad científica y género. Estudio del profesorado de una universidad española. Revista Mexicana de Sociología, 71(2), 351-386

Donsbach, W. (2006). The Identity of Communication Research. Journal of Communication, 56(3), 437-448. https://doi.org/10.1111/j.1460-2466.2006.00294.x

Durán-Bellonch, M y lon, G. (2014). ¿Investigadoras con éxito en la universidad...Cómo lo han logrado? Educación XX1, 17(1), 37-58. https://doi.org/10.5944/educxx1.17.1.10704

Escolano, E. (2006). Discriminación en un medio meritocrático: las profesoras en la universidad española. Revista mexicana de sociología, 68(2), 231-263. Recuperado de http://www.scielo.org.mx/scielo.php?script=sci_arttext\&pid=S018825032006000200002

Evans E. (2015) The Sisterhood: Inclusivity and Spaces. In: The Politics of Third Wave Feminisms. Gender and Politics Series. Palgrave Macmillan, London. https://doi.org/10.1057/9781137295279_6 
Gaitán, J. A y Piñuel, J. L (1998). Técnicas de investigación en comunicación social. Madrid, Síntesis.

Gill, R. (2006). Gender and the Media. Cambridge: Polity Press.

Gill, R. y Scharff, C. (Eds.) (2011). New Femininities: Postfeminism, Neoliberalism and Subjectivity. London: Palgrave Macmillan.

Giménez-Toledo, E. y Jiménez-Contreras, E. (2013). Black holes of communication scientific communication and meta-research. [Los agujeros negros de la comunicación: Comunicación científica y metainvestigación]. Comunicar, 41, 10-13. https://doi.org/10.3916/C41-2013-14

Gómez-Escalonilla, G. e Izquierdo-Iranzo, P. (2020). Contribuciones científicas presentadas en los congresos de la Asociación Española de Investigación de la Comunicación (2007-2018). El Profesional de la información, 29(4), e290440. https://doi.org/10.3145/epi.2020.jul.40

Gómez-Escalonilla, G. (2018). La investigación sobre la estructura de la comunicación en España. Comunicación presentada en el VI Congreso Internacional de la AE-IC “Comunicación y Conocimiento", Universidad de Salamanca, Salamanca, 26 al 29 de Junio, 2018.

Guil, A. (2007). Docentes e investigadoras en las universidades españolas: visualizando los techos de cristal. Revista de Investigación Educativa, 25(1), 11-31. Recuperado de https://revistas.um.es/rie/article/view/96561

Henry, A. (2004). Not My Mother's Sister: Generational Conflict and Third Wave Feminism. Bloomington: Indiana University Press.

Izquierdo-Iranzo, P., Gaitán-Moya, J. A. y Caffarel-Serra, C. (2020). Mapa de la mujer como sujeto y objeto de la investigación en comunicación en España. Comunicació. Revista de recerca i d'anàlisi, 37(1), 31-52. https://doi.org/10.2436/20.3008.01.189 
Kuhn, T. (1989). ¿Qué son las revoluciones científicas? Y otros ensayos. Barcelona: Paidós.

Lozano, I. e Iglesias, M. J. (2014). La presencia de las académicas a lo largo de la historia en la Educación Superior Española. Journal for Educators, Teachers and Trainers, 5(3), 204-216. Recuperado de http://hdl.handle.net/10045/40752

Martín Algarra, M., Serrano-Puche, J. y Rebolledo, M. (2018). La mujer en la investigación en comunicación en España: un análisis de la producción científica (20072013). adComunica, 15. http://dx.doi.org/10.6035/2174-0992.2018.15.5

Mendes, K., Ringrose, J. y Keller, J (2019). Digital Feminist Activism: Girls and Women Fight Back Against Rape Culture. Oxford: Oxford University Press. 